\title{
Analytic Hierarchy Process evaluation of English teaching quality in application-oriented universities
}

\author{
Liuanqi \\ Zhengzhou university of industrial technology.Henan.China
}

Keyword: Teaching quality;Analytic Hierarchy Process;Evaluation

\begin{abstract}
Teaching quality is the lifeblood of college survival and development, and improving the teaching quality is the eternal theme of teaching management. To establish teaching quality monitoring system is an important measure to guarantee improvement of the teaching quality. And the teaching quality evaluation is an effective way to improve the teaching quality. By reasonable teaching evaluation, the teaching management department can scientifically and comprehensively learn teachers' teaching situation. So that teachers can identify gaps and take measures to further facilitate the teaching. The method proposed in this paper is the evaluation of the teaching quality based on the dynamic Analytic Hierarchy Process sets. According to dynamic and Analytic Hierarchy Process teaching quality evaluation factors, this paper clarifies how to create dynamic Analytic Hierarchy Process sets and analyze the dynamic Analytic Hierarchy Process evaluation results. Meanwhile it also plays a valuable role in exploring better teaching quality evaluation methods.
\end{abstract}

\section{INTRODUCTION}

With the rapid development of language technology and improvement of a student's language literacy, a university student's ability is increasingly demanded in terms of language knowledge, skill and application in the information-based society. Because of the popularity of language education in recent years, English language courses are no longer limited to universities and colleges, but have begun to be applied in primary and middle schools. The economic condition of different regions, however, has led to an imbalance in language literacy.

The language knowledge level of students coming from developed regions or large and medium-sized cities has almost conformed to the teaching requirement of Englishs of languages, while a lot of students coming from remote areas or underdeveloped regions are unable to receive systematic language knowledge even though some of them have used languages before. Based on this condition, where the student's language operating level is unbalanced when they are enrolled at a university or college, it is imperative to implement AHP evaluation of English language course there .

AHP evaluation or teaching according to different levels, is a teaching method based on the theory of pedagogy and psychology. In AHP evaluation, students are divided into different levels according to their practical situation. By taking the national teaching syllabus as a basic target, teaching targets of different levels are formulated according to the specific situation, and are improved step-by-step.

Students are divided according to their levels and grades, and also according to their current levels of competence, so that a hierarchy of student and teaching objectives will be formed. In addition, according to their learning status, the system of upgrading and downgrading will be implemented as this is a highlight of AHP evaluation. For instance, Englishs of language is an obligatory course for all students.

If AHP evaluation is implemented, students will be required to take an examination in language operating skills at the beginning of their university/college life. Based on the academic performance in this examination, students will be divided into two grades (or levels) - an exempt programme or a compulsory programme, and students of each grade will be taught according to different teaching objectives in an effort to meet the requirements of application-oriented talent cultivation.

AHP evaluation, in fact, is affected by various factors, such as the characteristics of students, development and update of teaching methods, means and facilities, effective intervention of network teaching, etc. Drawing from successful experience of other universities and colleges, and in order to 
avoid the defects of over grading, the author has carried out reforms and practice on the AHP evaluation of the Englishs of language course based on the application-oriented guideline.

In addition, the author has also found effective solutions for the phenomenon where students with a good foundation cannot acquire enough knowledge and where students with a poor foundation fail to keep up with the teaching schedule. To further implement this teaching reform, the teaching of the English language course should be application-oriented and should focus on students of different levels.

\section{The determination method of subjective weight coefficient}

Using AHP to determine the subjective weights of evaluation indexes, the main steps are as follows:

Constructing judgment matrix, scaling method for evaluation of each factor to carry on the assignment, the importance of the judgment $\mathrm{j}$ matrix of structure are as follows:

The sheer level and a consistency check of judgment matrix. as the largest of judgment matrix eigenvalue and eigenvector, and carries on the satisfaction degree of validation. The calculation steps are following:

Calculated judgment matrix product of all the elements:

$$
m_{i}=\prod_{i=1}^{n} b_{i j} i=1,2, \cdots, n
$$

The Nth root:

$$
\varpi_{i}=\sqrt[n]{m_{i}}(i=1,2, \cdots, n)
$$

On vector normalization processing

$$
\varpi=\left(\varpi_{1}, \varpi_{2}, \cdots, \varpi_{n}\right)^{T},
$$

That is, to the desires of feature vector:

$$
\omega_{i}=\frac{\varpi_{i}}{\sum_{j=1}^{n} \varpi_{j}} i=1,2, \cdots, n
$$

Calculate the maximum characteristic root matrix:

$$
\lambda_{\max }: \lambda_{\max }=\frac{1}{n} \sum_{i=1}^{n} \frac{(A \omega)_{i}}{\omega_{i}}
$$

Consistency check of judgment matrix, namely $C R=C I / R I$, Among them, $C I=\frac{\lambda_{\max }-n}{n-1}$, When $C R<0.10$, the judgment matrix is satisfied consistency, otherwise we need readjust the element values.

Hierarchy total ordering. That needs to compute synthetic weight of each factor on the system 's overall goal, to determine the relative importance that all the factors of A-layer have for the system overall goal-A layer. This process has been run from the top layer to the bottom layer.

The method of determining the coefficients of objective weight. Entropy weight is a kind of objective method of endow with weight, namely to determine the index weight according to relative degree of change about the index's influence on the overall system, greater degree of relative change means larger weights of indicators[8-9]. The main steps of using Entropy weight to give weight are as follows:

The original matrix. Index of comprehensive evaluation of a sample about $m$, a problem about $n$ index, the formation of the original matrix are formatted as follows:

$$
X=\left\{x_{i j}\right\}_{m \times n}(0 \leq i \leq m, 0 \leq j \leq n)
$$

Index normalized processing. Since the dimension and the order of the magnitude of each indicator has certain differences, we have to eliminate the influences of different dimensions on the evaluation result, so it is necessary to standardize various indicators. 


$$
x_{i j}^{\prime}=\left\{\begin{array}{l}
\frac{x_{j}-x_{\min }}{x_{\max }-x_{\min }}-A \\
\frac{x_{\max }-x_{j}}{x_{\max }-x_{\min }}-B
\end{array}\right.
$$

Index after normalization treatment, the matrix proportion is as follows:

$$
Y=\left\{\frac{x_{i, j}^{\prime}}{\sum_{i=1}^{m} x_{i, j}^{\prime}}\right\}(0 \leq i \leq m, 0 \leq j \leq n)
$$

Calculation index information entropy and information utility value. The first item index $j$ of information entropy value is:

$$
e_{j}=-k \sum_{i=1}^{m} y_{i j} \ln y_{i j}(k=1 / \ln m)
$$

Information utility value depends on the difference in value between 1and the index of information entropy $e_{j}$. Its computation formula is:

$$
d j=1-e_{i}
$$

Calculation entropy of index. The entropy value of the $j$ item parameter values is defined as weight:

$$
\omega_{j}=\frac{d_{j}}{\sum_{i=1}^{m} d_{j}}
$$

First of all, Dividing factor set $U=\{u 1, u 2, \ldots, u m\} u i, i=1,2, \ldots, m$ into subset $U i=\{S i 1, S i 2, S \ldots$, $\operatorname{Sin}\} i=1,2, \ldots, S$ according to some properties so as to meet the conditions:

$$
\sum_{i=1}^{s} U_{i}=\operatorname{Sin} ; \bigcup_{i=1}^{s} U i ; U i \bigcap U i=\phi,(i \neq j)
$$

Then making a comprehensive evaluation on each factor set. If the evaluation result set $V=\{v 1$, $v 2, \ldots, v n\} v j, j=1,2, \ldots, n, i=1,2, \ldots$, Then, the distribution of the weight of $n$ is:

$$
W=\left\{w_{i 1}, w_{i 2}, \ldots, w_{i n}\right\} i, 1,2, \ldots, n
$$

Among them $\sum_{i=1}^{s}$ Win $=1,0 \leq$ Win $\leq 1$. If $R i$ is the single factor evaluation matrix, then we will get a vector:

$$
B i=W i \operatorname{Ri}=(b i 1, b i 2, \ldots \text { bim }) i=1,2, \ldots, s
$$

Then taking each $U i$ as a factor, $\mu=\Delta\{U 1, U 2, \ldots, U n\}$, Thus, $\mu$ is a set of factor, the single factor evaluation matrix of $\mu$ is:

$$
R_{j}=\left[\begin{array}{c}
B_{1} \\
B_{2} \\
\cdots \\
B_{3}
\end{array}\right]=\left[\begin{array}{cccc}
B_{11} & B_{12} & \cdots & B_{1 m} \\
B_{21} & B_{22} & \cdots & B_{2 m} \\
\cdots & \cdots & \cdots & \cdots \\
B_{s 1} & B_{s 2} & \cdots & B_{s m}
\end{array}\right]
$$

Each $U i$ is a part of $U$, which can reflect an attribute of $U$, it can be assigned according to the importance of the weight of $W j=\{w j 1, w j 2, \ldots W j n\} j=1,2, \ldots m$, therefore, we can get two grade evaluation vector:

$$
B j=W j \quad \circ R j=(B j 1, B j 2, \ldots, B j n)
$$

Calculation of index weight of degree of integration of tourism industry 
For weight calculation, it is mainly to invite experts to pairwise compare importance of various factors in each-level evaluation and the results are used to establish the judgment matrix of AHP weight distribution [10]. By consulting experts, this paper reaches A An judgment matrix and $A n \sim B$ judgment matrix of degree of integration of tourism industry in Hainan. Due to space limitations, this paper only describes A An judgment matrix.

Table 1. A An Judgment Matrix

\begin{tabular}{cccccc}
\hline $\mathrm{A}$ & $\mathrm{A}_{1}$ & $\mathrm{~A}_{2}$ & $\mathrm{~A}_{3}$ & $\mathrm{~A}_{4}$ & $\mathrm{~A}_{5}$ \\
\hline $\mathrm{A}_{1}$ & 1.00 & 0.20 & 0.20 & 0.14 & 0.33 \\
$\mathrm{~A}_{2}$ & 5.00 & 1.00 & 3.00 & 1.00 & 1.00 \\
$\mathrm{~A}_{3}$ & 5.00 & 0.33 & 1.00 & 1.00 & 1.00 \\
$\mathrm{~A}_{4}$ & 7.00 & 1.00 & 1.00 & 1.00 & 3.00 \\
$\mathrm{~A}_{5}$ & 3.00 & 1.00 & 1.00 & 0.33 & 1.00 \\
\hline
\end{tabular}

It is to calculate the feature vector of each judgment matrix as the weight of each criterion layer to goal level and to check consistency, the results are as follows: the weights of An criterion layer to goal layer A are $(0.05,0.29,0.19,0.31,0.17)$; the weights of $\mathrm{B} 1 \sim \mathrm{B} 5$ criterion layer to goal layer $\mathrm{A} 2$ are $(0.36,0.36,0.16,0.08,0.04)$; the weights of B6 B9 criterion layer to goal layer A2 are $(0.09,0.09,0.21,0.61)$; the weights of B10 B14 criterion layer to goal layer A3 are $(0.09,0.05$, 0.37, 0.07, 0.42); the weights of B15 B20 criterion layer to goal layer A4 are $(0.21,0.04,0.44,0.21$, $0.04,0.06)$; the weights of B21 B27 criterion layer to goal layer A5 are $(0.03,0.03,0.31,0.07$, $0.07,0.22,0.27$ ).

Table2. Results of Consistency Check Among Indexes

\begin{tabular}{cccccc}
\hline Matrix & $\lambda_{\text {max }}$ & C.I. & R.I. & C.R. & $\begin{array}{c}\text { Whether it } \\
\text { passed or } \\
\text { not }\end{array}$ \\
\hline $\mathrm{A}_{-} \mathrm{A}_{\mathrm{n}}$ & 5.28 & 0.07 & 1.12 & $0.06<0.1$ & Yes \\
$\mathrm{A}_{1}-\mathrm{B}_{5}$ & 5.14 & 0.03 & 1.12 & $0.03<0.1$ & Yes \\
$\mathrm{A}_{2}-\mathrm{B}_{9}$ & 4.15 & 0.05 & 0.89 & $0.06<0.1$ & Yes \\
$\mathrm{A}_{3}-\mathrm{B}_{14}$ & 5.14 & 0.03 & 1.12 & $0.03<0.1$ & Yes \\
$\mathrm{A}_{4}-\mathrm{B}_{20}$ & 6.48 & 0.10 & 1.26 & $0.08<0.1$ & Yes \\
$\mathrm{A}_{5}-\mathrm{B}_{27}$ & 7.43 & 0.07 & 1.36 & $0.05<0.1$ & Yes \\
\hline
\end{tabular}

As shown in the analytical statement and Tables 3-4, which report on academic performance, one can see that the language knowledge and operating ability that the students master before university are not as good as expected. To guarantee a satisfactory teaching result, only a few students can be exempted from taking the language courses. Most of the students obtained less than 30 points in the graded examination.

A sound performance was made in level-II examination with the average points of 84.6, with an excellent rate of $57.6 \%$ and an overall pass rate of almost $100 \%$. Due to the fact that the difficulty was greater in the final examination, the students only obtained the average score of 76.9, the excellent rate of $31.6 \%$, the pass rate of $91.6 \%$.

Table 3.Comparative analysis of scores section of grade 2014 language operating level test.

\begin{tabular}{|c|c|c|c|}
\hline Data item & $\begin{array}{c}\text { Entrance } \\
\text { examination }\end{array}$ & Level-II examination & $\begin{array}{c}\text { Final } \\
\text { examination }\end{array}$ \\
\hline $90-100$ points & 0 & 765 & 275 \\
\hline $80-90$ points & 0 & 903 & 771 \\
\hline $70-80$ points & 2 & 428 & 659 \\
\hline $60-70$ points & 15 & 156 & 411 \\
\hline $\begin{array}{c}\text { Below } 60 \\
\text { points }\end{array}$ & 2303 & 10 & 194 \\
\hline
\end{tabular}


Table 4.Cparative analysis of single item scores of grade 2014 language operating level test

\begin{tabular}{|l|c|c|c|}
\hline Data item & Entrance examination & Level-II examination & Final examination \\
\hline Average Point & 24.5 & 84.6 & 76.9 \\
\hline Highest Point & 72.9 & 99 & 99.2 \\
\hline Lowest Point & 2.1 & 19.3 & 13.5 \\
\hline
\end{tabular}

Table 5.Comparative analysis of the pass rate of grade 2014 language operating level test.

\begin{tabular}{|c|c|c|c|}
\hline Data item & Entrance examination & Level-II examination & Final examination \\
\hline Excellence rate & $0 \%$ & $57.60 \%$ & $31.60 \%$ \\
\hline Pass rate & $0.73 \%$ & $99.60 \%$ & $91.60 \%$ \\
\hline
\end{tabular}

\section{CONCLUSIONS}

After two years of experimenting with this system, the system that is divided into selective and compulsory courses, it has been found that AHP evaluation with cases is an effective teaching method. It has fully embodied the modern teaching philosophy that may vary from person to person, and that they should be taught accordingly. AHP evaluation also focuses on the demand of specialty, and should be combined with application-oriented and student-based individual development. As shown in the analysis of academic performance, the course teaching effect has been good and students have achieved a high level in language operation.Furthermore, the conduct of this research and the experimental results may provide a useful reference for the curriculum reform in similar universities and colleges.

\section{REFERENCE}

[1]. Hou GuangWen. Overview of Education Evaluation.HeBei:HeBei Education Press,1999

[2]. WuGang. Modern education evaluation basis.Shanghai:XueLin Press,1996,28.

[3]. Douwe,Beijaard and Nico Verloop, "Practical Knowledge, Studies in Educational Evaluation,” Assessing Teachers,1996, Vol. 22. No. 3, pp. 275-286.

[4]. Shen WeiXing, “ The University Teaching Quality Evaluation Item System,”ShangHai:DongHua University, 2003.

[5]. Liu Yao, “Quantizing Assessment about Teacher’s Business,”China Higher Education Evaluation,1998,2,62-64. 